\title{
CONGENITAL TRANSMISSION OF TRYPANOSOMA CRUZI IN EUROPE (SPAIN): A CASE REPORT
}

\author{
CRISTINA RIERA,* ANNA GUARRO, HOUSSEIN EL KASSAB, JOSÉ MARIA JORBA, MONTSERRAT CASTRO, \\ ROSER ANGRILL, MONTSERRAT GÁLLEGO, ROSER FISA, CARMEN MARTIN, ALEJANDRO LOBATO, AND \\ MONTSERRAT PORTÚS \\ Laboratori de Parasitologia, Facultat de Farmàcia, Universitat de Barcelona, Barcelona, Spain; Servei de Pediatria, Hospital \\ Residència Sant Camil, Sant Pere de Ribes, Barcelona, Spain; Servei d'Anatomia Patològica, Hospital Residència Sant Camil, Sant \\ Pere de Ribes, Barcelona, Spain; Servei de Microbiologia, Hospital Residència Sant Camil, Sant Pere de Ribes, Barcelona, Spain
}

\begin{abstract}
Here we report a documented case of congenital transmission of Trypanosoma cruzi from a Bolivian mother with chronic Chagas disease living in Spain. The serology and blood nested polymerase chain reaction (PCR) were positive for the mother, and amastigote forms were observed in histopathological study of the placenta and umbilical cord. Direct examination, culture, and nested PCR were positive in the blood of the neonate. At the age of 8 days, the neonate began treatment with $5-7.5 \mathrm{mg} / \mathrm{kg} /$ day of benznidazol, which was continued for 60 days. Direct examination, blood culture, and nested PCR were negative to T. cruzi 20 days after the start of treatment and remained negative 4 and 7 months thereafter. Serological tests were negative at 4 months. To detect congenital infection and initiate early treatment of infected newborns, protocols are required to detect Chagas disease in pregnant women who migrate from endemic to non-endemic areas.
\end{abstract}

\section{INTRODUCTION}

Chagas disease or American trypanosomiasis is a widely distributed endemic zoonosis in Central and South America, where $\sim 11$ million people are infected and an estimated 100 million people are at risk of infection. The disease is caused by the protozoan Trypanosoma cruzi and is usually considered as one affecting poor people in rural communities. In endemic areas, $T$. cruzi is transmitted mainly by triatomine insect vectors, which release excreta infected with the parasite into lacerated skin or mucosa. In addition to insect transmission, the infection may also occur through blood transfusion of whole blood or blood derivatives, congenital transmission from infected mothers, organ transplantation (kidney, heart, bone marrow, and others), and accidental contamination in the laboratory. ${ }^{1}$ Infection occurs to a lesser extent by oral transmission through food, such as meat, sugar cane juice, and fruit juice, contaminated with infected triatomines or their dejections. $^{1,2}$

At present, Chagas disease is a potential public health problem in Spain as a result of the increasing number of migrants from countries in which it is endemic. To our knowledge, a case of asymptomatic congenital transmission has been reported in Europe in a 5-year-old Latin American child living in Romania who had never traveled to an endemic area. ${ }^{3}$ Another congenital case has recently been diagnosed in a 2-year-old child in Barcelona (J. Muñoz and others, unpublished data). Here we report the first case of congenital Chagas disease transmission diagnosed in a neonate in Europe (Spain). We studied T. cruzi infection in the mother and the neonate, and a parasitological and serological longitudinal follow-up was performed on the child to monitor response to treatment.

\section{CASE REPORT}

A 28-year-old expectant Bolivian mother with chronic Chagas disease informed physicians of her condition at delivery.

* Address correspondence to Cristina Riera, Laboratori de Parasitologia, Facultat de Farmàcia, Universitat de Barcelona, Avda. Joan XXIII s.n., E-08028 Barcelona, Spain. E-mail: mcriera@ub.edu
The patient was asymptomatic, and anamnesis established that she had been infected at the age of 6 and diagnosed and treated at the age of 22 in Bolivia. The woman had two other children, 10 and 12 years of age, who lived in Bolivia and whose clinical condition was unknown.

The neonate was delivered at 37 weeks of gestation by caesarean section. Vaginal and rectal cultures for the mother were positive for Streptococus agalactiae, and she was treated with endovenous ampicillin during dilation. Caesarean delivery was required because attempts to induce birth failed. The newborn was a boy weighing $2.905 \mathrm{~kg}$. Preliminary examination of the infant detected no complications. The mother nursed the neonate, who showed a satisfactory evolution. Splenomegaly, hyperbilirubinemia, and encephalitis were not detected, and additional hematological and biochemical parameters were normal.

After delivery, the mother and the newborn were immediately examined for Chagas disease using parasitological and serological methods. The serological diagnosis of T. cruzi infection in sera was performed using a commercial ELISA with recombinant antigens (BioELISA Chagas; Biokit S.A., Lliça d'Amunt, Barcelona, Spain) and a non-commercial conventional ELISA with a whole lysate antigen obtained from epimastigotes of T. cruzi I strain. The bands recognized by the sera from mother and child were studied by Western blot (WB) analysis with the same antigen used in the conventional ELISA. A nested polymerase chain reaction (PCR) was performed following the standard technique (external primers TCZ1 and TCZ2 and internal primers TCZ3 and TCZ4, which amplify a DNA fragment of 149 bp of a repetitive sequence of nuclear DNA of $195 \mathrm{bp}){ }^{4}$

A blood sample from the mother was taken for laboratory diagnosis of Chagas disease. Microscopic observation of blood and culture in NNN medium were negative, and nested PCR was positive for T. cruzi DNA. Maternal seroreactivity to $T$. cruzi antigens was positive by ELISA and WB analyses (Table 1; Figure 1). Histopathological study of the placenta and umbilical cord was performed, and T. cruzi amastigote forms were observed in both samples (Figures 2 and 3).

Trypomastigote forms in the blood stream were detected using the microhematocrit concentration technique. The 
TABLE 1

Serological, molecular, and parasitological blood analysis of the mother and the newborn

\begin{tabular}{lcccc}
\hline & & & \multicolumn{2}{c}{ Newborn } \\
\cline { 3 - 5 } \multicolumn{1}{c}{ Methods } & Mother at & & \multicolumn{2}{c}{ Time after beginning treatment } \\
\cline { 3 - 5 } & delivery & At birth & 20 days & N months \\
Microscopy & Negative & Positive & Negative & Negative \\
Culture & Negative & Positive & Negative & Negative \\
Nested PCR & Positive & Positive & Negative & Negative \\
ELISA R* & 6.6 & 6.2 & 5.8 & 0.5 \\
ELISA W & 61 & 51 & 36 & 10 \\
\hline
\end{tabular}

* ELISA R (recombinant antigen, BioELISA Chagas, Biokit); cut-off $=1$

$\dagger$ ELISA W (whole antigen, in house): cut-off $=20$.

parasite was isolated by blood culture in NNN and T. cruzi DNA was detected by nested PCR. The strain was identified as $T$. cruzi I. Serological study by ELISA showed high levels

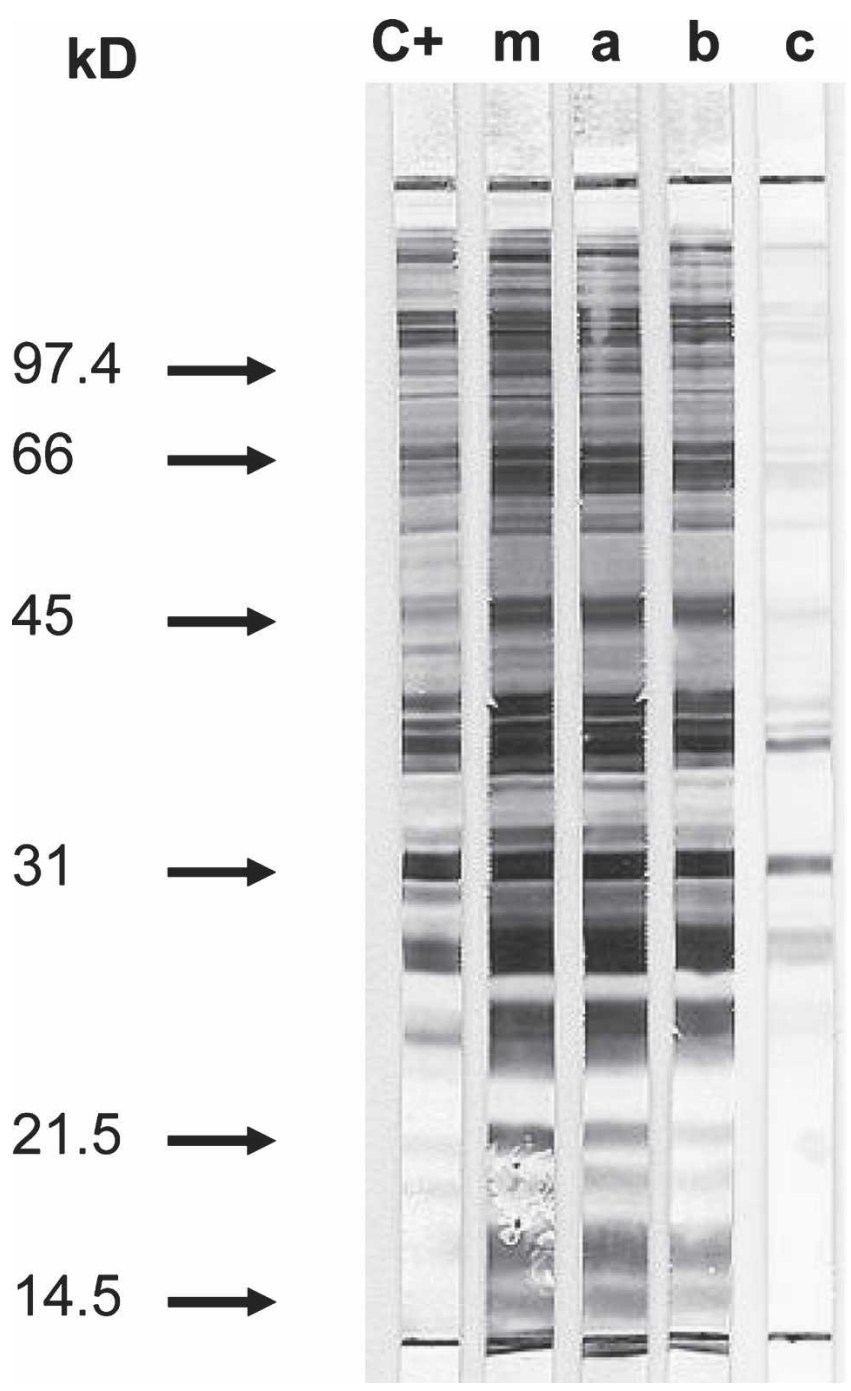

FIGURE 1. Trypanosoma cruzi antigen polypeptide recognition of sera from mother at delivery $(\mathrm{m})$ and newborn at 1 day (a), 20 days (b), and 4 months (c) after initiating anti-parasitic treatment. Whole antigen from $T$. cruzi epimastigotes was electrophoretically separated in sodium dodecyl sulphate-polyacrylamide gels (12\%) and transferred to nitrocellulose sheets. Immunoreaction was performed with sera at 1:50 dilution, and bound immunoglobulins were detected with protein A peroxidase conjugate (Sigma) at 1:1,000 dilution. of specific antibodies (Table 1), and the WB pattern of bands was identical in the newborn and mother (Figure 1).

Once congenital infection was confirmed, the neonate was treated with benznidazol (5-7.5 mg/kg/day) every 12 hours for 60 days. Parasitological and serological follow-up was performed at 20 days and 4 and 7 months after beginning treatment. Parasitemia, monitored by nested PCR and blood culture, was negative at day 20 after the start of treatment and remained negative at 4 and 7 months. At 4 months, ELISA tests were negative (Table 1 ), and the WB pattern showed a reduction in the number and intensity of bands (Figure 1). Clinical, hematological, and biochemical follow-up of the infant was performed for 12 months, during which time values were normal, and the child remained clinically asymptomatic. The infant showed good tolerance to treatment and normal development.

\section{DISCUSSION}

Chagas disease enters the chronic phase 2-4 months after the acute clinical symptoms disappear and generally develops

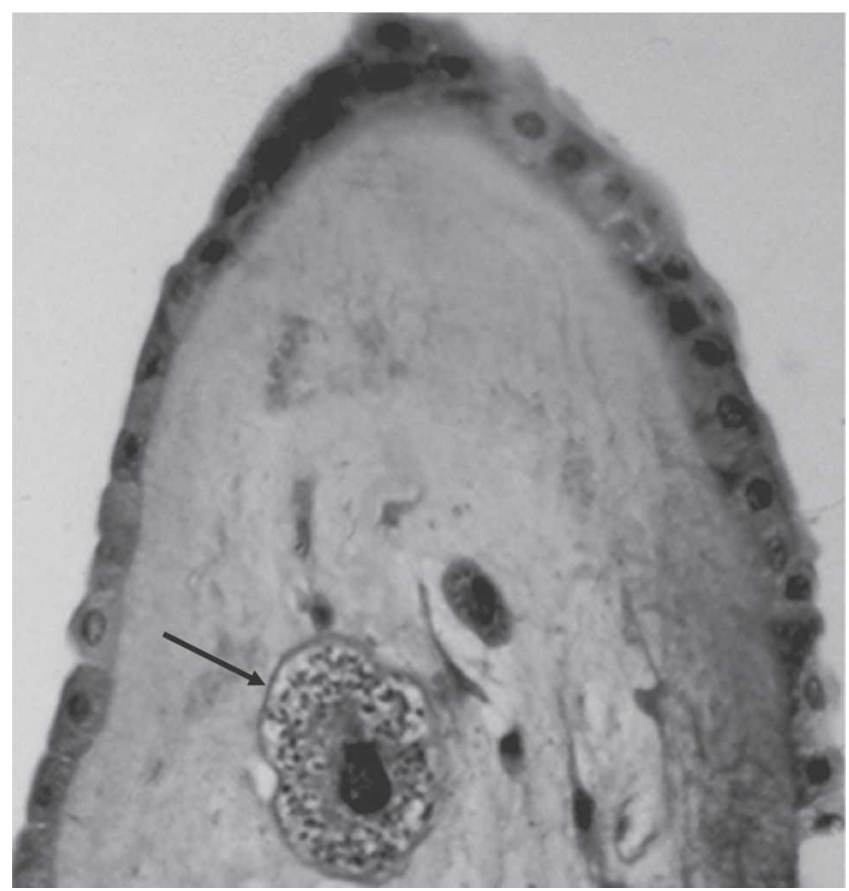

FIGURE 2. Histologic section of the extra-placental membranes, stained with hematoxylin-eosin, showing amastigotes of $T$. cruzi $(\mathrm{H} \& \mathrm{E}, \times 60)$. 


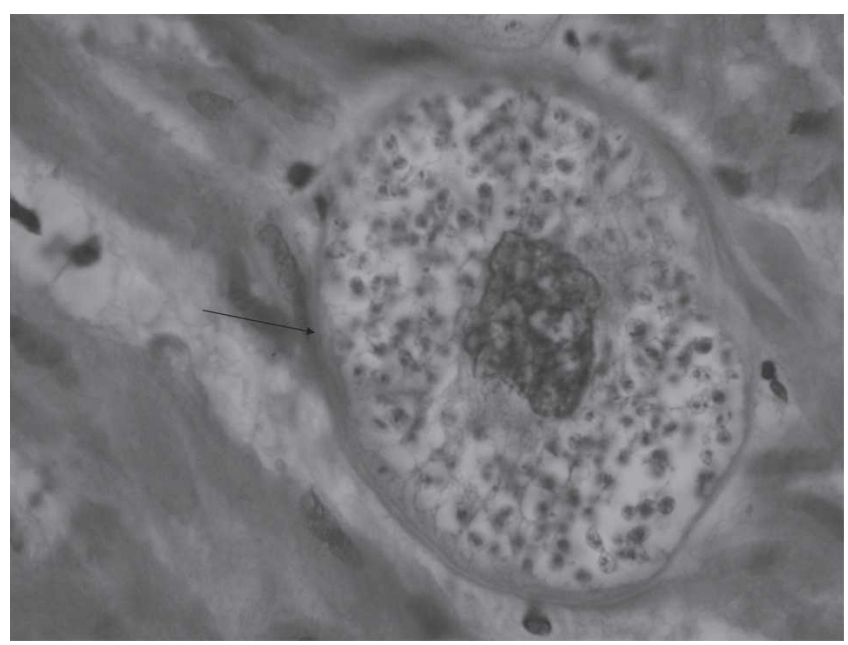

FIGURE 3. Histologic section of the umbilical cord, stained with hematoxylin-eosin, showing amastigotes of T. cruzi $(\mathrm{H} \& \mathrm{E}, \times 100)$.

with a long period of clinical latency called the indeterminate form, which lasts 10-30 years or throughout life. ${ }^{1}$ Infected pregnant women are usually asymptomatic, and the conditions and mechanisms of congenital transmission remain largely unknown. In endemic areas, seroprevalence in pregnant women may be as high as $81 \%$, and the probability of congenital transmission in children of chagasic mothers ranges from $<1 \%$ to $10 \%$, depending on the geographic area. ${ }^{5}$ Congenital transmission can occur in any phase of the maternal disease, but the risk is greater during the acute phase when parasitemia is intense and persistent. This period, however, is short, which might explain the small number of cases of transmission reported during this phase. ${ }^{6,7}$ In the chronic stage, there is intermittent parasitemia, and therefore, transmission may also occur, with rates estimated at around $1.6 \% .^{8,9}$ Indeed, the number of parasites in infected mothers, the strains involved, placental factors, and/or maternal immunity may determine congenital transmission of $T$. cruzi. ${ }^{10}$ Risk factors for the transmission of congenital Chagas disease and the pathophysiological and clinical features of congenital disease have been explained in several reports ${ }^{8,11-13}$ The diagnosis of congenital Chagas disease in the newborn is sometimes difficult because of low parasitemia and the persistence of positive serology, caused by the presence of antibodies transmitted to the neonate by the mother. In our case, the mother showed amastigote forms in the placenta and umbilical cord. Confirmation of T. cruzi in blood was positive only by nested PCR of the buffy coat, whereas the conventional parasitological techniques in blood were negative. Placental infection is not always synonymous with fetal infection, ${ }^{7}$ and no information is available on correlations between low parasitemia, detected by PCR, in the mother and the probability of fetal infection. In the case reported here, the detection of parasite by PCR in maternal blood together with the presence of parasite in placenta was associated with fetal infection.

Trypanosoma cruzi infection was diagnosed in the neonate by observation of trypomastigotes in smears from the buffy coat fraction (microhematocrit concentration) and by positive culture, thereby indicating the presence of circulating parasites. Twenty days after the neonate started treatment, direct examination, culture, and PCR were negative and remained negative during the follow-up.
ELISA detected high levels of IgG-specific antibodies in the newborn and in the mother. Nevertheless, high levels of $\mathrm{IgG}$ in the former has low predictive value of congenital transmission because of the passive transfer of maternal $\mathrm{IgG}$ antibodies, which could be present for up to 7 months in the non-infected child. ${ }^{14}$ Comparison of the immunologic profile of the mother and child, detected by WB analysis, allows differentiation between passively transmitted maternal antibodies and those newly synthesized in the infant, which are used in early postnatal diagnosis of congenital toxoplasmosis. ${ }^{15}$ In our case, no differences in the band pattern of the sera from the mother and newborn were detected after delivery, and no new bands were recognized by child sera during the follow-up period. Therefore, neonate production of antibodies could not be appreciated.

Microscopic observation of bloodstream trypomastigotes and/or culture of the buffy coat fraction of blood samples using the microhematocrit concentration technique are commonly used to diagnose congenital infection and are highly sensitive when parasitemia is high, as in the case reported here. In contrast, when parasitemia is low, conventional parasitological and serological techniques are not efficient enough to provide early diagnosis of congenital Chagas disease. However, PCR is an easy, rapid, and sensitive diagnostic method that requires only a small amount of blood, ${ }^{16}$ although unusual false-positive results have been described. ${ }^{17}$ In addition, this technique can be used to monitor parasitemia in infants after treatment. ${ }^{17,18}$

Early diagnosis of $T$. cruzi infection is essential for the rapid administration of anti-parasitic therapy. Studies on nifurtimox or benznidazol treatments show that the cure rate depends on the speed with which treatment is initiated. Most infants treated early test negative when examined 6 months to 2 years after starting treatment. ${ }^{12,17,18}$ The data obtained in our study are consistent with these results and show that treatment is effective when started during the first months of life.

Given the increasing number of women of fertile age migrating to Spain from regions with endemic Chagas disease, new cases of congenital transmission may occur. To increase the early detection of congenitally infected babies and thus facilitate their early treatment, we recommend screening for Chagas disease in pregnant women from endemic areas and the establishment of diagnostic protocols to detect the infection in the neonate.

\section{Received January 5, 2006. Accepted for publication July 5, 2006.}

Acknowledgments: The authors thank Dr. P. Bonay, Universidad Autónoma de Madrid, for genotyping $T$ cruzi strains. We also thank P. López-Chejade, M. Vergés, and S. Tebar (Laboratori de Parasitologia, Facultat de Farmàcia, Universitat de Barcelona) for providing technical assistance.

Authors' addresses: Cristina Riera, Montserrat Gállego, Roser Fisa, and Montserrat Portús, Laboratori de Parasitologia, Facultat de Farmàcia, Universitat de Barcelona, Avda. Joan XXIII s.n., E-08028 Barcelona, Spain, Telephone: 349340245 00, Fax: 349340245 04, E-mails: mcriera@ub.edu,mgallego@ub.edu,rfisa@ub.edu, mportus@ub.edu. Anna Guarro, Houssein El Kassab, José Maria Jorba, Carmen Martin, and Alejandro Lobato, Servei de Pediatria, Hospital Residència Sant Camil, Sant Pere de Ribes, Carretera Puigmoltó Km 0.8, Sant Pere de Ribes, 08810 Barcelona, Spain, Telephone: 349389600 25, Fax: 349389612 87, E-mails: santcamil@hrsantcamil.es,34578hk@comb.es, C.MEDIC@terra.es, santcamil@hrsantcamil.es. Montserrat Castro, Servei de Anatomia 
Patològica, Hospital Residència Sant Camil, Sant Pere de Ribes, Carretera Puigmoltó Km 0.8, Sant Pere de Ribes, 08810 Barcelona, Spain, Telephone: 349389600 25, Fax: 349389612 8, E-mail: mcastro@ava.scs.es. Roser Angrill, Servei de Microbiologia, Hospital Residència Sant Camil, Sant Pere de Ribes, Carretera Puigmoltó Km 0.8, Sant Pere de Ribes, 08810 Barcelona, Spain, Telephone: 3493896 00 25, Fax: 349389612 8, E-mail: rangrill@cli.scs.es.

\section{REFERENCES}

1. Prata A, 2001. Clinical and epidemiological aspects of Chagas disease. Lancet Infect Dis 1: 92-100.

2. Benchimol Barbosa PR, 2006. The oral transmission of Chagas' disease: An acute form of infection responsible for regional outbreaks. Int J Cardiol.

3. Pehrson PO, Wahlgren M, Bengtsson E, 1981. Asymptomatic congenital Chagas' disease in a 5-year-old child. Scand J Infect Dis 13: 307-308.

4. Marcon GE, Andrade PD, de Albuquerque DM, Wanderley da Silva J, de Almeida EA, Guariento ME, Costa SC, 2002. Use of a nested-polymerase chain reaction (N-PCR) to detect Trypanosoma cruzi in blood samples from chronic chagasic patients and patients with doubtful serologies. Diag Microbial Infect Dis 43: 39-43.

5. Bittencourt AL, 2000. Transmissiao vertical da doença de Chagas. Brener Z, Andrade ZA, Barral-Netto M, eds. Trypanosoma cruzi e doença de Chagas. Second edition. Rio de Janeiro: Editora Guanabara Koogan SA, 16-20.

6. Okumura M, Aparecida dos Santos V, Camargo ME, Schultz R, Zugaib M, 2004. Prenatal diagnosis of congenital Chagas' disease (American trypanosomiasis). Prenat Diagn 24: 179-181.

7. Moretti E, Basso B, Castro I, Carrizo Paez M, Chaul M, Barbieri G, Canal Feijoo D, Sartori MJ, Carrizo Paez R, 2005. Chagas' disease: Study of congenital transmission in cases of acute maternal infection. Rev Soc Bras Med Trop 38: 53-55.

8. Bittencourt AL, 1992. Possible risk factors for vertical transmission of Chagas' disease. Rev Inst Med Trop Sao Paulo 34: 403-408.

9. Reiche EM, Inouye MM, Bonametti AM, Jankevicius X, 1996. Congenital Chagas disease: Epidemiology, laboratorial diagnosis, prognosis and treatment. J Pediatr 72: 125-132.
10. Hermann E, Truyens C, Alonso-Vega C, Rodriguez P, Berthe A, Torrico F, Carlier Y, 2004. Congenital transmission of Trypanosoma cruzi is associated with maternal enhanced parasitemia and decreased production of interferon-gamma in response to parasite antigens. $J$ Infect Dis 189: 1274-1281.

11. Hernandez-Matheson IM, Frankowski RF, Held B, 1983. Foetomaternal morbidity in the presence of antibodies to Trypanosoma cruzi. Trans R Soc Trop Med Hyg 77: 405.

12. Blanco SB, Segura EL, Cura EN, Chuit R, Tulian L, Flores I, Garbarino G, Villalonga JF, Gurtler RE, 2000. Congenital transmission of Trypanosoma cruzi: An operational outline for detecting and treating infected infants in north-western Argentina. Trop Med Int Health 5: 293-301.

13. Torrico F, Alonso-Vega C, Suarez E, Rodriguez P, Torrico MC, Dramaix M, Truyens C, Carlier Y, 2004. Maternal Trypanosoma cruzi infection, pregnancy outcome, morbidity, and mortality of congenitally infected and non-infected newborns in Bolivia. Am J Trop Med Hyg 70: 201-209.

14. Moya P, Moretti E, Basso B, Blanco S, Sanmartino C, de Cura AS, 1989. Enfermedad de Chagas neonatal, diagnóstico de laboratorio en el primer año de vida. Medicina (B Aires) 49: 595-599.

15. Robert-Gangneux F, Commerce V, Tourte-Schaefer C, DupouyCamet J, 1999. Performance of a Western blot assay to compare mother and newborn anti-Toxoplasma antibodies for the early neonatal diagnosis of congenital toxoplasmosis. Eur $J$ Clin Microbiol Infect Dis 18: 648-655.

16. Virreira M, Torrico F, Truyens C, Alonso-Vega C, Solano M, Carlier Y, Svoboda M, 2003. Comparison of polymerase chain reaction methods for reliable and easy detection of congenital Trypanosoma cruzi infection. Am J Trop Med Hyg 68: 574582.

17. Schijman AG, Altcheh J, Burgos JM, Biancardi M, Bisio M, Levin MJ, Freilij H, 2003. Aetiological treatment of congenital Chagas' disease diagnosed and monitored by the polymerase chain reaction. $J$ Antimicrob Chemother 52: 441-449.

18. Russomando G, de Tomassone MM, de Guillen I, Acosta N, Vera N, Almiron M, Candia N, Calcena MF, Figueredo A, 1998. Treatment of congenital Chagas' disease diagnosed and followed up by the polymerase chain reaction. Am J Trop Med Hyg 59: 487-491. 